\title{
La importancia del poder de policía municipal, en la provincia de Buenos Aires, respecto a las fumigaciones con agroquímicos
}

\author{
Juan Ignacio Moreno ${ }^{1}$ \\ Universidad Nacional de La Plata - Argentina
}

Revista Derechos en Acción ISSN 2525-1678/ e-ISSN 2525-1686

Año 5/Nº 14, Verano 2019-2020 (21 diciembre a 20 marzo), 697-714

DOI: https://doi.org/10.24215/25251678e372

ORCID: https://orcid.org/0000-0002-9516-3456

\section{I. ¿Cuáles son las consecuencias de la agricultura industrial?}

Para afrontar las consecuencias en la aplicación de agroquímicos y brindar una respuesta jurídica, resulta trascendental acompañar el debate junto a una serie de hechos relevantes que constatan su peligrosidad para la salud humana y el ambiente.

En el año 2017, la Redactora Especial sobre el derecho a la alimentación presentó un informe ${ }^{2}$ ante el Consejo de Derechos Humanos de Naciones Unidas respecto a las consecuencias de los agroquímicos para los derechos humanos. Se manifestó que los agroquímicos se promovieron de forma agresiva peligrando un verdadero goce del derecho humano a la alimentación. El

\footnotetext{
Abogado (UNLP). Ayudante alumno por concurso de la materia Derecho Agrario Cátedra 3 de la Facultad de Ciencias Jurídicas y Sociales (UNLP). Investigador (FCJyS-UNLP)

Contacto: ignacio_moreno@live.com.ar

2 InformedelaRedactoraEspecialdeNacionesUnidassobreelderechoalaalimentacióndelaño 2017. https://www.refworld.org/cgibin/texis/vtx/rwmain/opendocpdf.pdf?reldoc=y\&docid= 58ad94864
} 
informe recepta unas 200.000 muertes por intoxicación aguda ante la exposición a los agroquímicos.

El avance tecnológico gestado por las grandes potencias implicó la masificación en la producción de alimentos. En este sistema productivo se pierde la fertilidad de los suelos a causa del constante incremento de insumos químicos sobre la tierra que generan una reducción de sus nutrientes. Es decir, que mediante la utilización de los agroquímicos los productores requieren una mayor cantidad de tierra para mantener su nivel de producción. Por el fin mercantilista, los pequeños y medianos productores se endeudan adquiriendo insumos dolarizados (fabricados por multinacionales como Bayer-Monsanto, Syngenta, Dupont y Baff).

Retomando el Informe de la Redactora, este sistema productivo no logró eliminar el hambre del mundo y la dependencia a los insumos químicos acarreó una alimentación inadecuada.

En base a esta agricultura industrial se ha generado un trastorno sobre el ecosistema del cual todos somos parte. Este aspecto se percibe ante la desaparición de la biodiversidad a razón de las fumigaciones corrompiendo la cadena alimentaria entre las especies de animales depredadoras y sus presas.

En el marco de un derecho humano a la alimentación, los Estados deben exigir una seguridad alimentaria real y que los alimentos estén libres de plaguicidas. También se debe garantizar los derechos a los sectores más vulnerables, sujetos pasivos, frente a estas prácticas como:

- Los trabajadores rurales, quienes tienen una exposición cotidiana con estos tóxicos al utilizarlos mediante la aplicación terrestre en tractores, manualmente con las mochilas o incluso exponiéndose a ser bañados literalmente por esta lluvia tóxica emanada de avionetas.

- Las comunidades agrícolas, comprendida por los pobladores que residen en zonas próximas a la actividad agraria.

- Los niños, los cuales según la Organización Internacional del Trabajo (OIT) trabajan en la agricultura de todo el 
mundo en un $60 \%$ comprendidos entre los 5 y 17 años que equivale a 98 millones de niñas y niños ${ }^{3}$. A su vez, la exposición de agroquímicos sobre la infancia involucra problemas en el desarrollo intelectual ${ }^{4}$.

- Mujeres embarazadas que viven en zonas aledañas a estas producciones intensivas y han sufrido abortos espontáneos tras las fumigaciones.

La Red de Acción en Plaguicidas, una organización sin fines de lucro, estipuló que entre 1 millón y 41 millones de personas son afectadas anualmente por la exposición a plaguicidas 5 . Por esta constante exposición a los agroquímicos, destaca la Redactora, se los ha vinculado al cáncer, Alzheimer, Parkinson, trastornos hormonales, a problemas de desarrollo y a esterilidad. También se lo asocia con alergias y efectos neurológicos como pérdida de memoria. Cabe considerar que los síntomas pueden no presentarse de una manera inmediata a la fumigación y observarse sus efectos con el transcurso de los meses.

Otro aspecto que incide en la propagación de las consecuencias de los agroquímicos se concentra en la falta de control, en algunos casos porque se carece de los recursos humanos necesarios para abarcar todo el territorio de una localidad. Dicho control, materializado en el poder de policía municipal, se torna fundamental cuando el glifosato, un herbicida de gran utilización en la Argentina, ha sido considerado, en el año 2015, por la Organización Mundial de la Salud (OMS) como probable cancerígeno humano y que cuenta con resoluciones judiciales que comprueban esta situación. El glifosato hace 10 años, según expone el Dr. Damián Marino, se aplicaba en unos 3 litros por

\footnotetext{
3 Trabajo Infantil en la Agricultura por la Organización Internacional del Trabajo. Disponible en: https://www.ilo.org/ipec/areas/Agriculture/lang--es/index.htm

4 Eyhorn, Reducing Pesticide Use, p. 9. Disponible en: https://pdfs.semanticscholar.org/e51 e/12f5491b11820f10119911ee480857464e74.pdf Último acceso 24/10/19.

5 Red de Acción en Plaguicidas, Communities in Peril: Global Report on Health Impacts of Pesticide Use in Agriculture (2010). Disponible en: http://www.pan-germany.org/download/ PAN-I_CBM-Global-Report_1006-final.pdf Último acceso 22/10/19
} 
hectárea mientras que en la actualidad se aplican 15 litros por hectárea ${ }^{6}$ denotando un incremento a razón de la expansión del agronegocio.

Finalmente, un mito que es importante derribar consiste que "las verduras y frutas con residuos tóxicos pueden con un simple lavado quedar limpios de los mismos". Los plaguicidas se aplican sobre las raíces de los cultivos, como en lechugas o frutas, por lo cual su contenido químico se propaga por toda la planta. Irremediablemente aquellos desechos químicos llegarán a nuestras mesas.

Frente a la vulneración de los derechos humanos (considerados dentro de este concepto la salud, alimentación y el ambiente) que viven los pueblos fumigados se abordará, a continuación, el término de "poder de policía" como herramienta esencial para hacer frente a este suceso.

\section{II. ¿Qué se entiende por poder de policía?}

Con el fin de obtener una mayor precisión sobre el poder de policía resulta indispensable comprender los términos de poder y policía de una manera independiente para luego analizar su conjunción y vinculación con la fumigación de agroquímicos.

Dentro del derecho público el poder es concebido de manera única y se divide en funciones (como la legislativa, administrativa y jurisdiccional) las cuales son ejercidas por órganos del Estado. (Gordillo, 2013)

Desde la edad antigua hasta el siglo XV se vinculaba al concepto de policía con las actividades estatales. Luego el término de policía redujo su amplitud y se abocó a la actividad administrativa interna como reglamentación, poder de coacción y dictado de órdenes. Estas actividades vislumbraban a

6 Nota del medio Noticiauno del día 5/6/2018 bajo el título "Argentina lidera el ranking mundial por la cantidad de glifosato que usa el campo" Disponible en: http://www. noticiauno.com.ar/nota/3502-Argentina-lidera-el-ranking-mundial-por-la-cantidad-deglifosato-que-usa-el-campo Último acceso 25/10/2019 
la policía como una función del Estado y en particular como una función ejecutiva. En el siglo XVII se sostenía que esta intervención estatal, en el marco del poder de policía, debía ejercerse cuando el buen orden de la comunidad se encontrara en peligro para garantizar la libertad y la seguridad mediante el poder de coacción.

Desde esta visión liberal, el poder de policía tiene la facultad de imponer límites y restricciones a los derechos individuales con el fin de proteger la seguridad, salubridad y moralidad. En cambio, el Estado de bienestar modifica este paradigma y entiende al poder de policía como la complementación de la prevención del daño y la promoción del bien común. Por lo tanto, esta definición establece una relación indispensable entre prevenir un daño, como en el caso del ambiente, donde consecuentemente se estará eliminando peligros y evitando perturbaciones al orden con la promoción de un bienestar común para una vida digna de los seres humanos.

\section{Legislación sobre el poder de policía ambiental}

La reforma constitucional del año 1994 incorporó la protección de derechos colectivos que atañen a todos los ciudadanos. El que aquí nos incumbe es la protección al ambiente que establece el deber de garantizar a las generaciones presentes un ambiente sano y equilibrado con una ética intergeneracional (art.41 Constitución Nacional, 1994). A través de esta ética se exige cuidar y preservar el ambiente para que las generaciones futuras puedan disfrutarlo en las mismas condiciones que las generaciones presentes. Se establece la responsabilidad por parte del Estado para prevenir daños al ambiente.

El actual modelo del agronegocio, más allá de su rentabilidad inmediata, no colabora con este designio constitucional ya que se ponen en riesgo los recursos de las generaciones presentes y futuras mediante la aplicación de un sistema de agricultura intensivo basado en el monocultivo y la reducción de la biodiversidad. Además, la satisfacción de las necesidades 
es realmente discutible, ¿Qué tipo de alimentación se fomenta con la agricultura industrial? ¿Una alimentación que contenga residuos tóxicos? ¿Un consumo de carne sostenida por medicamentos a consecuencia del encierro del ganado en los feedlot? Ante estos interrogantes es trascendental un ejercicio efectivo del poder de policía para evitar las vulneraciones a derechos humanos fundamentales de las generaciones presentes y futuras que habitan y habitarán la República Argentina.

Continuando con la norma ambiental, el Congreso de la Nación tiene la facultad de dictar normas de presupuestos mínimos, es decir, un marco regulatorio que establezca un conjunto de bases mínimas sobre las cuales se conserve los recursos naturales del país.

En el año 2002 el Congreso de la Nación aprobó la Ley General de Ambiente 25675 complementándose lo dispuesto por el texto constitucional. La ley trata los principios que rigen el ámbito del derecho ambiental de los cuales se puede destacar al precautorio y preventivo. En el caso del preventivo, se comprende que el derecho ambiental busca preservar el bien colectivo siendo este bien un elemento de funcionamiento social (Cafferata, 2004). Es decir que los seres humanos nos encontramos dentro de un entorno que el Estado tiene el deber de protegerlo por su aspecto público de los recursos y que afecta directa o indirectamente a toda la comunidad.

Desde el ámbito de la provincia de Buenos Aires y en consonancia con el marco protectorio nacional sobre ambiente, en el año 1994 se incorporó su protección. En su art.28, el Estado provincial garantiza el goce a un ambiente sano y su protección para las generaciones futuras. También desarrolla el dominio originario, no delegado a la Nación, de los recursos naturales en su territorio mientras este aprovechamiento sea mediante una gestión ambientalmente adecuada. Esta gestión consiste en la preservación, recuperación y conservación de dichos recursos para evitar la degradación del ecosistema. Este artículo constitucional se instrumentó a través de la Ley $n^{\circ} 11723$ Integral de Medioambiente y los Recursos Naturales que contiene un 
procedimiento administrativo sobre el impacto ambiental de los proyectos que produzcan algún efecto negativo en el ambiente y/o sus recursos naturales.

Mediante este plexo normativo se establece la conservación del ambiente, por parte del Estado, desde su preservación como su recomposición. En el caso de la aplicación de agroquímicos, la protección al ambiente, se materializar a través de la ley provincial 10699 donde los municipios (art.16 ley 10699), con la estructura necesaria, tendrán la facultad de ejercer junto al Ministerio de Agroindustria de la provincia de Buenos Aires el poder de policía. Este poder de policía municipal se consolida mediante ordenanzas que restringen la utilización de agroquímicos en zonas urbanas, en escuelas rurales, en cauces de agua y lugares que afecten derechos humanos de los pobladores. Es importante anticipar que se requiere de una política común entre municipio y provincia para lograr un verdadero bienestar social ante esta dramática situación de las fumigaciones.

\section{El clamor social da sus frutos}

En este espacio se tratará la acción u omisión del poder de policía, sobre el uso de agroquímicos, en las localidades bonaerenses de Mercedes, San Antonio de Areco y Pergamino. Como primer acercamiento se vislumbra un reclamo de los pobladores que irrumpe en la agenda de los gobernantes.

Los pueblos fumigados se levantan e interpelan a sus representantes políticos en defensa por una vida digna. Ante este reclamo social es fundamental un verdadero compromiso político para detener los abusos del agronegocio.

\section{Localidad de Mercedes}

En el año 2011, mediante la ordenanza No 6998/2011, el Concejo Deliberante de la ciudad de Mercedes reguló la aplicación de agroquímicos. En dicha normativa se precisó los derechos fundamentales que se protegían, desde su primer artículo, 
como el ambiente, la salud y los recursos naturales frente a este sistema productivo. La ordenanza delimita las zonas de protección frente a las fumigaciones, que a continuación se desarrollarán.

El artículo 4 de la presente ordenanza disponía la prohibición de fumigaciones aéreas con una distancia de 300 metros respecto a las viviendas del Partido de Mercedes y con previo aviso de 48hs al dueño de la vivienda. Durante el año 2013, fue modificado el art. 4 a través de la ordenanza $N^{\circ} 7217 / 13$ estableciéndose una prohibición para toda fumigación aérea en una distancia no menor a $2 \mathrm{~km}$. de centros poblados sin poder sobrevolarlos con o sin carga. Dicha reforma brinda una mayor protección legal para la salud de los habitantes de Mercedes y en consonancia con el decreto 499/91 (artículo 38) que reglamentó la ley provincial sobre agroquímicos 10699.

En su art. 5 se prohíbe la aplicación terrestre con equipos autopropulsados (conocidos como "mosquitos") en las áreas urbanas del municipio y efectuarse a una distancia de 500 metros de las áreas urbanizadas y zonas de población consolidada.

Sobre las zonas donde se encuentren establecimientos escolares, centros primarios de salud, reservas naturales se delimita una distancia de 100 metros a su alrededor y donde se prohíbe utilizar equipos manuales (denominados "mochilas") para aplicar agroquímicos. Entre un perímetro de 100 a 300 metros se permite fumigar con mosquitos y en ambas situaciones dar aviso por escrito a la autoridad del lugar con 48 horas de anticipación.

También limita la aplicación de agroquímicos sobre áreas linderas a cursos de agua y caminos rurales sean limítrofes o internos a los campos en un radio de 100 metros. Los mosquitos no podrán circular por centros urbanos salvo por las rutas nacionales y provinciales y en caso de extrema necesidad circularán sin carga, limpios y con los picos cerrados para evitar el goteo.

Para un mayor control la ordenanza dispone la creación de un registro de aplicadores de agroquímicos para las fumigaciones 
aéreas y terrestres bajo la reglamentación de la Dirección de Salud del Municipio de Mercedes. Las transgresiones a esta normativa por fumigación terrestre serán sancionadas por el Código de Faltas y procederán decomisos de productos y equipos y en caso de reiteradas infracciones se lo sancionará con la máxima cantidad de módulos de multa (90 módulos) y se lo quitará del Registro de aplicadores con aviso a la Dirección Provincial de Fiscalización Agropecuaria y alimentaria. Por último, las violaciones de las prohibiciones de las fumigaciones aéreas serán penadas con una multa de 2000 módulos y enviando una comunicación a la Dirección Provincial actuante.

Esta normativa busca brindar una protección integral a la problemática de la aplicación de agroquímicos. Se determinan los límites para fumigaciones aéreas y terrestres. Además, se exige a los aplicadores la inscripción en el registro local y poseer la receta agronómica (exigencia presente en la normativa provincial). A pesar de esto continúa resultando escasa la protección sobre fumigaciones terrestres por una limitación inferior a otras localidades.

El 23/10/17 vecinos de Mercedes alertaron a la policía rural sobre un tractor sin identificación dispuesto a fumigar. Cuando la Patrulla Rural realizó las averiguaciones constató que el aplicador no poseía la receta agronómica ni el certificado de inscripción en el Registro de Aplicadores de la Municipalidad. Además, sin el previo aviso a los vecinos lindantes con $48 \mathrm{hs} \mathrm{de}$ anticipación como lo establece la ordenanza municipal. El mosquito contenía una combinación de Glifosato y otro producto químico que se encontraba prohibido para aplicarse en aquel período del año. En esta situación se percibe como el ejercicio del poder de policía municipal precisa la colaboración de los vecinos, quienes alertan estas actividades ilícitas que vulneran las ordenanzas locales.

Lamentablemente las consecuencias del agronegocio continúan haciendo estragos como sucedió el 27 de septiembre del año 2019 cuando se suscitó un voraz incendio en un depósito 
de agroquímicos en la localidad de Mercedes ${ }^{7}$. Nuevamente el gobierno provincial de María Eugenia Vidal mantuvo silencio y se demoró el accionar del O.P.D.S. para contrarrestar el impacto de esta explosión de químicos sumamente dañinos para la salud humana.

\section{Localidad de San Antonio de Areco}

Otra ciudad bonaerense afectada por las fumigaciones ha sido la ciudad de San Antonio de Areco que contó con un reclamo popular y tuvo una respuesta política.

Para garantizar el derecho a la salud se requiere gozar plenamente de un ambiente sano. Por lo tanto, las políticas públicas son esenciales en protección de toda la comunidad.

Por este motivo, con impulso del intendente Francisco Durañona, se modificó la ordenanza 3919/14 que regula la aplicación de agroquímicos. Bajo la ordenanza $4226 / 17^{8}$, atento al incremento de muertes a razón de enfermedades oncológicas y respiratorias por la contaminación de las fumigaciones en la localidad bonaerense se decidió prohibir las fumigaciones aéreas en San Antonio de Areco. Este relevamiento de casos fue realizado por médicos de la Universidad Nacional de Rosario arrojando que el cáncer es el problema de salud más resonante con un $40 \%$ y las fuentes de contaminación se asocian al río contaminado y cerealeras.

Los residuos de los agroquímicos quedan presentes en el suelo y en el agua. Sus consecuencias toman relación con el daño ambiental cuyo nexo es una causalidad circular, es decir, que los elementos que lo integran interactúan donde la causa precede al efecto como el efecto a la causa (Falbo, 2009). Claramente las

\footnotetext{
7 Nota del medio Radio Gráfica del día 20/10/2019 bajo el título “Mercedes víctima del agronegocio" (Disponible en https://radiografica.org.ar/2019/10/20/mercedes-victima-delagronegocio/ Último acceso 7/11/2019)

8 Ordenanza 4226/17 que establece la prohibición de fumigaciones aéreas en San Antonio de Areco. (Disponible en: http://instituciones.areco.gob.ar/hcd/index.php/2017/ 4379-4226-17-regulacion-aplicacion-agroquimicos-aerea)
} 
distintas causas de contaminación se retroalimentan y agravan el daño hacia la salud de los pobladores.

En el mismo año 2017 un grupo de aplicadores solicitó una medida cautelar frente a esta ordenanza y fue rechazada por el Juzgado Contencioso Administrativo $\mathrm{N}^{\mathrm{O}} 1$ del Departamento Judicial de Mercedes manteniendo la vigencia de la ordenanza 4226/17. En dicho fallo se destacó el poder reglamentario municipal en materia ambiental como un deber irrenunciable y cuya facultad es concurrente con la provincia. Un verdadero reconocimiento al poder de policía ambiental correspondiente a los municipios.

Finalmente es oportuno destacar el compromiso de la docente Ana Zabaloy, fallecida en el año 2019, quien se dedicó a crear la Red Federal de Docentes por la Vida ${ }^{9}$. Incluso cuando regresaba a su casa fue fumigada por un mosquito y aspiró accidentalmente 24-D (sustancia química de agroquímicos) causándole una parálisis facial. Como resaltaba Ana Zabaloy "los docentes rurales son testigos directos del costo humano basado en transgénicos y venenos"10.

\section{Localidad de Pergamino}

La ciudad de Pergamino ha atravesado un camino judicial destacable frente a la vulneración de la salud de sus habitantes. Ha resultado fundamental la celeridad de las autoridades judiciales ante el reclamo social en la producción de pruebas científicas, sobre el agua, como al momento de brindar una respuesta

9 Para una mayor información sobre la vida de Ana Zabaloy y las posturas de distintos referentes políticos, se recomienda la lectura del artículo realizado por los periodistas Julieta Bugacoff y Federico Muiña. (Disponible en: https://www.elcohetealaluna.com/ catorce-miligramos-de-glifosato-ensangre/?fbclid=IwAR280pWSb8snWw59xUkhx9SiRTjGP wgMk3hqg3S6ZuDWkVWyLmAD0b32pU Último acceso 20/12/2019)

10 Testimonio de la docente Ana Zabaloy sobre las consecuencias de los agroquímicos. En la misma manifiesta la problemática sobre la sociedad, los temores para realizar denuncias, el peligro constante en la exposición para los niños, el rol del Estado como la importancia de instalar la temática en las escuelas rurales. Entrevista realizada por el medio "Huerquen" (Disponible en: https://www.youtube.com/watch?v=54G9IceaR2E\&t=1s Último acceso 20/12/2019) 
limitando las fumigaciones y exigiendo su cumplimiento. Por esta razón es conveniente realizar un breve recorrido por la historia judicial que aconteció en la localidad.

En abril del año 2019 se detectaron la presencia de agroquímicos en el agua potable que consumían los pobladores de la ciudad de Pergamino ${ }^{11}$. En un principio constado por especialistas del INTA Balcarce, CONICET y el Dr. Damián Marino (UNLP) pero que ante un intento infundado de la autoridad del agua bonaerense sobre el rigor de esta prueba, el juez Carlos Villafuerte Ruzo (Titular del Juzgado Federal de Primera Instancia en lo Criminal y Correccional $\mathrm{N}^{\circ} 2$ de San Nicolás) solicitó al equipo toxicológico de la Corte Suprema de Justicia de la Nación que con nuevas muestras ratificó el trabajo de precedente y confirmó la presencia de 18 agroquímicos en el agua resultando un riesgo para la salud.

Con estos resultados, en el mes de agosto de 2019 se ordenó ampliar la medida cautelar del mes de abril de 600 metros por vía terrestre extendiéndose la prohibición a la totalidad de la ciudad de Pergamino con un límite de exclusión de 1095 metros para aplicaciones terrestres y de 3000 metros para las aéreas ${ }^{12}$. Además, se ordenó la suspensión de autorizaciones de aplicaciones futuras en la ciudad. Cabe destacar que la ordenanza 8126/14 en su art.6 dispone una exclusión para fumigaciones de 100 metros del área urbana.

\footnotetext{
11 Este reclamo ha sido impulsado por un colectivo conocido como "Madres de Barrios Fumigados de Pergamino". Sabrina Ortiz decidió estudiar Derecho y al recibirse iniciar los reclamos judiciales al experimentar las consecuencias de estas fumigaciones (tuvo la pérdida de un embarazo tras ser fumigada por una avioneta y tanto sus hijos como ella se les detectó glifosato en sangre). Para mayor información entrevista a Sabrina Ortiz para Radio La Tosca (Disponible en: https://ar.radiocut.fm/audiocut/entrevista-a-sabrina-ortiz-fundadoramadres-barrios-fumigados-pergamino/_)

12 Esta resolución tuvo como precedente científico un informe del equipo de investigación de Genotoxicidad de la Universidad de Río Cuarto a cargo de la Dra. En Biología Delia Aiassa que demostró la posible consecuencia de daños genéticos para las personas que tuvieran residuos de agroquímicos en sangre. Disponible en: https://infocielo.com/nota/109058/ fallo-historico-la-justicia-federal-prohibio-las-fumigaciones-a-3-mil-metros-en-pergamino/ Último acceso 20/12/2019.
} 
En octubre del 2019, la Cámara Federal de Rosario rechazó el planteo del Municipio de Pergamino para ser querellante en esta causa. Adujo que el municipio como persona jurídica de carácter público no podría ser imputado en esta causa, pero la presunta responsabilidad de funcionarios del municipio era suficiente para desestimar el pedido.

Durante noviembre, una comitiva del Departamento de Delitos Ambientales de la Policía Federal detuvo a un propietario de un predio rural, un ingeniero agrónomo y arrendatario del campo y al aplicador de agroquímicos por orden del Juzgado Federal $\mathrm{N}^{\circ} 2$ de San Nicolás tras violar medidas precautorias establecidas por la justicia respecto a la prohibición para fumigar. Se les imputaba la aplicación de agroquímicos a 90 metros de viviendas familiares y establecimientos educativos.

Ante esta situación, el juez Carlos Villafuerte Ruzo dispuso allanamientos en el Centro de Atención Primaria de la Salud (CAPS) de Villa Alicia y el Hospital Interzonal General de Agudos "San José". La finalidad de estas diligencias, efectuada por efectivos de la policía federal, consistió en obtener historias clínicas y constancias de familias afectadas por la contaminación del agua en los barrios Villa Alicia, Luard Kayard y La Guarida dependientes a la localidad de Pergamino. Además, se había brindado una línea telefónica para realizar denuncias frente a incumplimientos por parte de fumigadores.

Este accionar proactivo de los jueces debe ser entendido entorno a la grave vulneración de derechos colectivos que aquejan a una población. Desde ya una sentencia no podrá confrontar un modelo agroexportador que brinda soluciones inmediatas en la economía argentina pero si resultar revelador de sus consecuencias hacia la calidad de vida.

Recientemente Raúl Eugenio Zaffaroni expuso en el Vaticano $^{13}$ que las sentencias son un acto de gobierno porque los

13 Exposición de Raúl Eugenio Zaffaroni, en el mes de junio de 2019, en el marco de un Congreso sobre "Derechos sociales y doctrina franciscana" en el Vaticano. (Disponible en: https://www.youtube.com/watch?v=YVeMqCnu9fY Último acceso 17/12/2019) 
jueces son parte de la sociedad y no pueden ser apolíticos frente a una problemática tan visible en nuestros días. Por ende, las sentencias tienen un valor simbólico más allá de su ejecución.

\section{Conclusiones}

Como expresa el art.16 de la ley provincial 10699, tendrán la potestad de ejercer el poder de policía aquellos municipios que posean la estructura necesaria. Diversos municipios por la carencia de recursos económicos y humanos se encuentran imposibilitados para realizar un fehaciente poder de policía y controlar, luego de dictar una ordenanza apropiada, la aplicación de agroquímicos.

Por ende, no solo es necesario un compromiso total de los municipios sino que se requiere una cooperación por parte del gobierno provincial, es decir, que ambos poderes se dirijan hacia la protección de la salud de los habitantes.

En este aspecto el gobierno de María Eugenia Vidal fijó una postura a favor del agronegocio y avanzó en consecuencia vulnerando derechos humanos de los bonaerenses.

El 6 de julio del 2016, fue aprobado por el Senado de la provincia de Buenos Aires el proyecto para reformar la ley agroquímicos en el cual se delimitaban las fumigaciones, pero en contraposición a los límites determinados por la Suprema Corte de Justicia de Buenos Aires (la SCBA en el fallo D.JE.F. s/ acción de amparo" y "Picorelli" había fijado una distancia de 1000 metros para fumigaciones terrestres ${ }^{14}$ ). En este proyecto de ley se modificaba el término de agroquímicos por fitosanitarios en un claro mensaje ideológico buscando quitarle al término una connotación negativa. Además, era un texto poco preciso donde no se establecían los distintos tipos de agroquímicos

\footnotetext{
14 La jurisprudencia provincial ha sido abordada, anteriormente, en el trabajo al cual remito, Moreno, J. I. (2019). Agroquímicos: su impacto en la legislación y jurisprudencia bonaerense. Derechos En Acción, 12(12), 319. https://doi.org/10.24215/25251678e319 Disponible en: https://revistas.unlp.edu.ar/ReDeA/article/view/8742
} 
según su grado de toxicidad como si lo establece el art.7 de la ley 10699 (este artículo clasifica los agroquímicos según los de uso y venta libre, los de venta profesional y los de uso registrado). El texto era un claro retroceso para la protección de la salud porque prohibía fumigaciones terrestres a 100 metros del centro urbano y aéreas a 500 metros de la zona urbana cuando la ley 10699 limita esta actividad aérea a $2 \mathrm{~km}$. También determinaba, irrisoriamente, un límite de 10 metros de zonas urbanas la fumigación terrestre de plaguicidas de clases III y IV (en esta categoría ingresa el glifosato). Finalmente, este proyecto no tuvo tratamiento en la cámara de diputados provincial.

Tras este intento fallido, en enero de 2019 se promulgó la resolución ministerial 246/18 del ministro de agroindustria Leonardo Sarquis (ex gerente de la división Semillas de Monsanto Argentina durante 3 años), donde se establecían zonas de amortiguamiento para la no aplicación de agroquímicos, pero sin especificar distancias mínimas. Es decir que esta resolución era aún más aberrante que el proyecto de ley del año 2016 ya que acentuaba la vaguedad y ambigüedad del texto y permitía que se fumigara sobre escuelas rurales fuera del horario escolar. Frente al reclamo social y amparos presentados por distintos organismos la resolución fue suspendida por el plazo de un año.

Esta postura provincial fue acompañada por el gobierno nacional de Mauricio Macri cuando en abril del 2019 trató de irresponsable ${ }^{15}$ un fallo de la Sala III de la Cámara Civil y Comercial de Paraná (Provincia de Entre Ríos) que anuló los artículos 1 y 2 del decreto del gobernador Gustavo Bordet que permitían fumigar a 100 metros terrestres y a 500 metros aéreos de una escuela rural. A través de esta valiosa jurisprudencia se dejó sin efecto, en aquel entonces, estas distancias

\footnotetext{
15 Nota del medio Página 12 del día 4/4/2019 bajo el título “Macri defendió el uso de agrotóxicos sin control". Disponible en: https://www.pagina12.com.ar/185254-macridefendio-el-uso-de-agrotoxicos-sin-control último acceso 11/11/2019.
} 
y se restableció la vigencia de las distancias anteriores a este decreto de 1.000 metros para aplicaciones terrestres y 3.000 metros para aplicaciones aéreas como la prohibición de fumigar con agroquímicos en horario escolar. Luego tras la derrota en las elecciones presidenciales del mes de octubre del 2019, el presidente Macri dispuso por el decreto 740/19 una quita generalizada de fondos de entidades del Estado y unos 8100 millones de pesos del Instituto Nacional de Tecnología Agropecuaria (INTA) para compensar la reducción del IVA. Otra medida vinculada con achicar el Estado y perjudicar la información y formación sobre la producción agropecuaria de uno de los organismos más federales del país.

Frente a esta postura provincial y nacional es sumamente valiosa la postura que han decidido tomar ciertos municipios como el de San Antonio de Areco y buscar garantizar el derecho a una vida digna y atender al reclamo de sus habitantes. Desde ya este ejercicio de poder de policía se obstaculiza a través de la desfinanciación nacional de organismos como el INTA vinculado a la concientización sobre prácticas agrícolas. En definitiva, quienes terminan ejerciendo el control, pero sin la estructura estatal, de estas aplicaciones de agroquímicos son los vecinos al denunciarlos a los municipios y ante la justicia.

Ha llegado la hora de los pueblos y resulta irrisorio negar la realidad. La jurisprudencia ha oído el reclamo social con fallos ejemplares como en el caso de la SCBA o el precedentemente destacado en la provincia de Entre Ríos. Esta búsqueda de justicia ha sido acompañada por informes e investigaciones de campo por distintas universidades del país y no se pueden tener puntos grises en esta problemática de salud. Frente a la colisión de intereses por gobiernos de turno resplandece la marcha de los pueblos fumigados que continuarán alzando la voz ante el avasallamiento de los derechos humanos. En este camino hacia la justicia será imprescindible contar con un compromiso político que tenga como causa, la causa del pueblo y no intereses personales y empresariales. 


\section{Referencias Bibliográficas}

Cafferata N. (2004), "Introducción al Derecho Ambiental", Secretaría de Medio Ambiente y Recursos Naturales y otros, México.

Eyhorn, Reducing Pesticide Use, p. 9. Disponible en: https://pdfs. semanticscholar.org/e51e/12f5491b11820f10119911ee 480857 464e74.pdf Último acceso 24/10/19.

Falbo A. (2009), Derecho Ambiental, Librería Editora Platense, La Plata.

Gordillo A. (2013), "Tratado de Derecho Administrativo y obras selectas: Teoría general del Derecho Administrativo, tomo 8", Fundación de Derecho Administrativo, Buenos Aires.

Moreno, J. I. (2019). Agroquímicos: su impacto en la legislación y jurisprudencia bonaerense. Derechos En Acción, 12(12), 319. https://doi.org/10.24215/25251678e319 Disponible en: https://revistas.unlp.edu.ar/ReDeA/article/view/8742

Ordenanza Disponible en: http://www.mercedes.gob.ar/ docabiertos/wp-content/uploads/2015/01/6998_2011.pdf Último acceso 29/10/19

Red de Acción en Plaguicidas, Communities in Peril: Global Report on Health Impacts of Pesticide Use in Agriculture (2010). Disponible en: http://www.pan-germany.org/download/PAN-I_ CBM-Global-Report_1006-final.pdf Último acceso 22/10/19

Trabajo Infantil en la Agricultura por la Organización Internacional del Trabajo. Disponible en: https://www.ilo.org/ipec/areas/ Agriculture/lang--es/index.htm

\section{Artículos}

Nota del medio Hoy Mercedes, del día 25/10/2017, bajo el título "Patrulla Rural impidió fumigaciones en campos de Mercedes"(Disponible en: https://www.hoymercedes.com.ar/ principal/2017/10/25/patrulla-rural-impidio-fumigaciones-encampos-de-mercedes/ Último acceso 29/10/19)

Nota del medio Noticiauno del día 5/6/2018 bajo el título "Argentina lidera el ranking mundial por la cantidad de glifosato que usa el campo" (Disponible en: http://www.noticiauno.com.ar/ nota/3502-Argentina-lidera-el-ranking-mundial-por-la-cantidad-de-glifosato-que-usa-el-campo Último acceso 25/10/2019) 
Nota del medio Radio Gráfica del día 20/10/2019 bajo el título "Mercedes víctima del agronegocio (Disponible en https:// radiografica.org.ar/2019/10/20/mercedes-victima-del-agronegocio/ Último acceso 7/11/2019)

Nota del medio Página 12, del día 4/4/2019, bajo el título "Macri defendió el uso de agrotóxicos sin control". Disponible en: https://www.pagina12.com.ar/185254-macri-defendio-el-usode-agrotoxicos-sin-control Último acceso 11/11/2019.

Nota del medio "El Cohete a la Luna", realizada por Julieta Bugacoff y Federico Muiña, del día 13/10/2019, bajo el título "14 miligramos de glifosato en sangre" (Disponible en: https://www. elcohetealaluna.com/catorce-miligramos-de-glifosato-ensangr e/?fbclid=IwAR28QpWSb8snWw59xUkhx9SiRTjGPwgMk3hqg 3S6ZuDWkVWyLmADOb32pU Último acceso 20/12/2019)

\section{Videoteca}

Entrevista realizada por el medio "Huerquen" a la docente rural Ana Zabaloy. (Disponible en: https://www.youtube.com/watch? $\mathrm{v}=54 \mathrm{G} 91 \mathrm{ceaR2E} \& \mathrm{t}=1 \mathrm{~s}$ Último acceso 20/12/2019)

Exposición de Raúl Eugenio Zaffaroni, en el mes de junio de 2019, en el marco de un Congreso sobre "Derechos sociales y doctrina franciscana” en el Vaticano. (Disponible en: https://www.youtube.com/watch?v=YVeMqCnu9fY Último acceso 17/12/2019) 